ESAIM: PROCEEDINGS AND SURVEYS, February 2015, Vol. 49, p. 23-36

Samira EL YACOUBI, Larbi AFIFI, El Hassan ZERRIK, Abdessamad TRIDANE, Editors

\title{
REGIONAL STABILITY AND STABILITY RADIUS*
}

\author{
A. Bernoussi ${ }^{1}$ And A. Bel FeKiH ${ }^{2}$ \\ Dedicated to Abdelhaq El Jai for his 65th birthday.
}

\begin{abstract}
In this work we consider the problem of stability, for distributed parameter systems, through the space variable. We give an extension of the stability radius, introduced by A. J. Pritchard and S. Townley $[7,10]$, to the regional case. This consists to determine the "smallest disturbance" which destabilizes regionally an exponentially stable system. We prove in particular that for a certain given class of distributed parameter systems, it is possible to destabilize regionally an exponential stable system without destabilizing it totally.

Résumé. Nous considérons dans ce travail le problème de la stabilité pour les systèmes à paramètres distribués avec une variable espace. Nous donnons une extension au cas régional du rayon de stabilité, introduit par A. J. Pritchard et S. Townley [7,10]. Il s'agit de déterminer la "plus petite perturbation" qui déstabilise régionalement un système exponentiellement stable. Nous montrons en particulier que pour certaines classes de systèmes distribués données, il est possible de déstabiliser régionalement un système initialement exponentiellement stable sans pour autant le déstabiliser totalement.
\end{abstract}

Keywords: Distributed parameter systems, regional robust stability, total instability, stability radius.

\section{INTRODUCTION}

Stability is one of the most important concepts introduced in analysis and control of systems. An unstable system has practical applications if we can stabilize it and the stability/stabilizability will be more interesting if the system remains stable even in the presence of some disturbances (robust stability/robustness). For distributed parameter systems, characterized by a spatiotemporal evolution, the recent works developed by A. El Jai and his team show the importance of space variable in the study of such systems. Indeed, in [1], it was introduced for distributed parameter systems, a notion more appropriate for highlighting the spatial aspect in the analysis and control of such systems: It is the regional analysis. That consists to study the system (analysis and/or control) no more globally (on the whole field $\Omega$ where is defined the system) but only regionally (on a certain region $\sigma \subset \Omega$ ). These concepts of regional controllability/observability and stability/stabilizability were introduced. It has been shown that it is possible to control (observe or stabilize) regionally some systems which are not globally controllable (observable or stabilizable) $[1,12-14,18]$.

In this work we consider the problem of robust regional stability. In particular we consider the problem of determining the regional stability radius (for a given region $\sigma \subset \Omega$ ). Indeed, for the stability radius, A. J.

* This work is supported by Académie Hassan II des Sciences et Techniques in Morocco.

${ }^{1}$ GAT team , Faculty of Sciences and Techniques B.P. 416, Tangier, Morocco. (a.samed.bernoussi@gmail.com)

2 Team of Mathematical Modeling and Control. Faculty of Sciences and Techniques B.P. 416, Tangier, Morocco. (abelfekih@gmail.com)

(C) EDP Sciences, SMAI 2015 
Pritchard and S. Townley $[7,10]$ have considered the problem of determining the smallest disturbance which destabilizes a given exponentially stable system on $\Omega$. But since there are some systems which are regionally exponentially stable without being globally exponentially stable, we consider the following problems:

(1) For an exponentially stable system the smallest disturbance which destabilizes the system could destabilize it on a very particular region $\sigma \subset \Omega$ (without destabilizing the other regions) ? If yes which one ? and for what type of systems?

(2) For an exponentially stable system can we define a regional stability radius for each region? If yes this means that each region $\sigma$ admits its proper stability radius $r_{\sigma}$. In this case are there any relationships between $r_{\sigma}$ and the global stability radius $r_{\Omega}$ ? Also can we compare the degree of stability (of such a regions) and determine which region is the most vulnerable to instability ? [3,5]

(3) For two regions $\sigma_{1}$ and $\sigma_{2}$, can the destabilization of the one of them cause the destabilization of the other?

This paper is organized as follow: In the first section we recall the stability radius. In the second section we recall the regional stability definition as it was introduced by El jai et al. [1], [11] and we introduce the "total instability" concept. In the third section we introduce the regional stability radius. Some examples are given to illustrate the results.

\section{STABILITY RADIUS}

We begin by recalling the definition of the stability radius as it was given in [7].

Let $\Omega$ be an open and bounded subset of $\mathbb{R}^{n}$ representing the geometric field in which evolves the system described by the following state equation

$$
\left\{\begin{array}{l}
\dot{z}(t)=A z(t)+B u(t) \quad t>0 \\
z(0)=z_{0}
\end{array}\right.
$$

with the measure function

$$
y(t)=C z(t)
$$

where $A$ is a differential operator with domain $D(A)$, self-adjoint and with a compact resolvent and generates a strongly continuous semigroup $(S(t))_{t \geq 0}$ on the state space $X . C \in \mathcal{L}(\underline{X}, Y)$ and $B \in \mathcal{L}(U, \bar{X})$.

$X, U$ and $Y$ are separable Hilbert spaces. Generally $U$ and $Y$ designate respectively state and observations spaces. $\underline{X}$ and $\bar{X}$ are Banach spaces considered in a way to consider the case where $B$ and/or $C$ are unbounded.

To determine the stability radius of a given system, the choice of the nature of the disturbance is very important. Indeed in [6] Desh and Schappacher considered a class of disturbance of $A$ in the form $T=$ $A(I+P Q)+L Q$ while in [7] Pritchard and Townley have considered disturbance of $A$ in the form $A+B D C$.

In this work we consider the stability radius as it was been introduced by Pritchard and Townley in [7].

For this we consider a disturbance of the system (1) in the form $u=D y$, where $D \in \mathcal{L}(Y, U)$. The system (1) is written as

$$
\left\{\begin{array}{l}
\dot{z}=A z+B D C z \\
z(0)=z_{0}
\end{array}\right.
$$

Under some assumption (which will be clarified later), the solution of the disturbed system is given by

$$
z(t)=S(t) z_{0}+\int_{0}^{t} S(t-s) B D C z(s) \mathrm{d} s
$$

Which gives

$$
y(t)=C z(t)=C S(t) z_{0}+C \int_{0}^{t} S(t-s) B D C z(s) \mathrm{d} s
$$


and

where $y_{0}=C S(t) z_{0}$ and

$$
y=y_{0}+C L D y
$$

$$
L: \mathrm{L}^{2}(0, \infty ; U) \rightarrow \mathrm{L}^{2}(0, \infty ; X)
$$

is defined by

$$
(L u)(t)=\int_{0}^{t} S(t-s) B u(s) \mathrm{d} s \quad t>0
$$

For $D \in \mathcal{D}$ where $\mathcal{D}$ is a subset of $\mathcal{L}\left(L_{Y}^{2}, L_{U}^{2}\right)$.

Assume that the system $(A, B, C)$ is in the Pritchard-Salamon class i.e. the following assumptions are satisfied:

1. $X \subset X \subset \bar{X}$ with a dense and continuous injections.

2. $S(t)$ extends (restricted) to a strongly continuous semigroup on $\underline{X}(\bar{X})$.

3. The domain of $A$ in $\bar{X}$ is a subset of $\underline{X} ; D_{\bar{X}}(A) \subset \underline{X}$.

4. There exists $M, \alpha>0$ such that $\|S(t)\| \leq M e^{-\alpha t}, t \geq 0$. on the three spaces $\underline{X}, X$ and $\bar{X}$.

5. For all $T>0$ there exists $k$ such that for all $x \in \underline{X},\|C S(.) x\|_{\mathrm{L}^{2}(0, T ; Y)} \leq k\|x\|_{X}$.

6. For all $T>0$, there exists $k_{T}$ such that for all $u(.) \in \mathrm{L}^{2}(0, T ; U), \int_{0}^{T} S(s) B u(s) \mathrm{d} s \in X$ and

$$
\left\|\int_{0}^{T} S(s) B u(s) \mathrm{d} s\right\|_{X} \leq k_{T}\|u(.)\|_{\mathrm{L}^{2}(0, T ; U)}
$$

7. There exists $k$ such that for all $u(.) \in L_{U}^{2}$,

$$
\left\|\int_{0} S(.-s) B u(s) \mathrm{d} s\right\|_{L_{\underline{X}}^{2}} \leq k\|u(.)\|_{L^{2}(U)}
$$

Then the solution of (3) is given by

$$
z(t)=S(t) z_{0}+\int_{0}^{t} S(t-s) B D C z(s) \mathrm{d} s
$$

We have the following definition [7]

Definition 1.1. The stability radius of system (3) is the greatest positive real $\mathrm{r}$, noted $r(A, B, C)$ defined by $r(A, B, C)=\sup \{r>0$ such that if $\|D\|<r$, then

the solution of (3) is exponentially stable for all $z_{0}$ in $X$ \}

This means that the stability radius is the smallest disturbance which destabilizes an exponentially stable system. We have the following result [7]:

Theorem 1.2. If the system is in the Pritchard-Salamon class and $\sup _{\omega}\|G(i \omega)\|<+\infty$ then

$$
r(A, B, C)=\frac{1}{\|C L\|}=\frac{1}{\sup _{\omega}\|G(i \omega)\|}
$$

where $G(i \omega)$ is the transfer function of system $(S)$ and $L: \mathrm{L}^{2}(0, \infty ; U) \rightarrow \mathrm{L}^{2}(0, \infty ; Y)$ is defined by (4).

For more details see [7]. 


\section{REGIONAL STABILITY / TOTAL INSTABILITY}

\subsection{Regional stability}

Let $\sigma$ be a given region, with non null measure (meas $(\sigma) \neq 0$ where meas $(\sigma)$ is the Lebesgue measure of $\sigma$ ), fixed in $\Omega$ and $\chi_{\sigma}$ the characteristic function of $\sigma$. We have the following definition [1]:

\section{Definition 2.1.}

(1) A semigroup $(S(t))_{t \geq 0}$ is said to be $\sigma$-regionally exponentially (or $\sigma$-exponentially) stable on $X=\mathrm{L}^{2}(\Omega$ ) if there exists two strictly positive constants $M_{\sigma}$ and $\alpha_{\sigma}$ such that

$$
\left\|\chi_{\sigma} S(t)\right\| \leq M_{\sigma} e^{-\alpha_{\sigma} t} \quad, \quad t \geq 0
$$

(2) The system (1) is said to be regionally exponentially stable on $\sigma$ (or $\sigma$-exponentially stable) if the semigroup $(S(t))_{t \geq 0}$ generated by the operator $A$ is $\sigma$-exponentially stable on $\mathrm{L}^{2}(\Omega)$.

(3) We say that the system is $\sigma$-exponentially unstable if it is not $\sigma$-exponentially stable.

If $(S(t))_{t \geq 0}$ is a semigroup $\sigma$-exponentially stable on $\mathrm{L}^{2}(\Omega)$ then for all $z_{0} \in \mathrm{L}^{2}(\Omega)$ the solution of the associated autonomous system (1) satisfies

$$
\left\|\chi_{\sigma} z(t)\right\|=\left\|\chi_{\sigma} S(t) z_{0}\right\| \leq\left\|\chi_{\sigma} S(t)\right\|\left\|z_{0}\right\| \leq M_{\sigma} e^{-\alpha_{\sigma} t}\left\|z_{0}\right\|
$$

and then $\lim _{t \rightarrow+\infty}\left\|\chi_{\sigma} z(t)\right\|=0$.

\section{Remark 2.2.}

(1) In this paper we consider only the case where the measure of $\sigma$ is non null. ( $\operatorname{meas}(\sigma) \neq 0$ ).

(2) An exponentially stable (globally) system (on $\Omega$ ) is regionally exponentially stable on all region $\sigma \subset \Omega$ (with $\bar{\sigma} \subset \Omega$ and meas $(\sigma) \neq 0$ ) but the converse is not true as it is shown in the following example:

Example 2.3. Consider the system given by the following state equation:

$$
(S) \begin{cases}\dot{z}(t)=(x-4) z(t) & , \quad x \in] 0,5[, \quad t>0 \\ z(x, 0)=z_{0}(x) & x \in] 0,5[ \\ z(0, t)=z(5, t)=0 & t>0\end{cases}
$$

For all $a \in] 0,4[$ this system is exponentially stable on a $\sigma=] 0, a[$ but it isn't exponentially stable on $\Omega=] 0,5[$.

$$
z(x, t)=e^{(x-4) t} z_{0}(x) \quad 0<x<5
$$

then

$$
\begin{gathered}
\left.S(t) z_{0}: x \in\right] 0,5\left[\rightarrow e^{(x-4) t} z_{0}(x)\right. \\
\left\|\chi_{\sigma} S(t) z_{0}\right\|^{2}=\int_{0}^{a}\left|e^{(x-4) t} z_{0}(x)\right|^{2} \mathrm{~d} x \leqslant e^{2(a-4) t} \int_{0}^{a} z_{0}(x)^{2} \mathrm{~d} x \leqslant e^{2(a-4) t} \int_{0}^{5} z_{0}(x)^{2} \mathrm{~d} x \\
\leq M_{\sigma} e^{-\alpha_{\sigma} t} \quad, \quad t \geq 0
\end{gathered}
$$

$M_{\sigma}=1$ and $\alpha_{\sigma}=2(4-a)$. then the system is not exponentially stable because for $\left.z_{0}(x)=\chi_{] 4,5[}(x), x \in\right] 0,5[$, we have $\left\|z_{0}\right\|=1$ and

$$
\left\|S(t) z_{0}\right\|^{2}=\int_{4}^{5}\left|e^{(x-4) t}\right|^{2} \mathrm{~d} x=\frac{e^{2 t}-1}{2 t}=\frac{e^{2 t}-1}{2 t}\left\|z_{0}\right\|^{2}
$$


then

and then the semigroup is not exponentially stable.

$$
\|S(t)\|^{2} \geqslant \frac{e^{2 t}-1}{2 t} \quad, \quad \forall t>0
$$

In the following proposition we summarize some elementary results [1].

Proposition 2.4. Let $\sigma_{1}$ and $\sigma_{2}$ be two regions of $\Omega$, with meas $\left(\sigma_{j}\right)>0, j=1,2$; Then:

(i): If the system is exponentially stable on $\sigma_{2}$ and $\sigma_{1} \subset \sigma_{2}$, then the system is exponentially stable on $\sigma_{1}$.

(ii): If the system is exponentially stable on $\sigma_{1}$ and on $\sigma_{2}$, then it is stable on $\sigma_{1} \cup \sigma_{2}$ and on $\sigma_{1} \cap \sigma_{2}$ (if meas $\left.\left(\sigma_{1} \cap \sigma_{2}\right)>0\right)$.

\subsection{Instability and total instability}

As we consider in this work the problem of determining the regional stability radius i. e. the "smallest disturbance" which destabilizes regionally a given system, we consider in this subsection the problem of "instability" through space variable. We have the following definitions

Definition 2.5. The system $(S)$ is said to be totally exponentially unstable if for all subset $\sigma$ of $\Omega$ such that meas $(\sigma) \neq 0,(S)$ is $\sigma$-exponentially unstable.

A totally exponentially unstable system is globally (on $\Omega$ ) exponentially unstable. The converse is not true (see example 2.3). However there exists many systems for which we have the equivalence. Then we have the following definition

Definition 2.6. The system $(S)$ is said to be of UTU type (Unstable/Totally Unstable) if: $(S)$ is exponentially unstable if and only if $(S)$ is totally exponentially unstable.

Definition 2.6 means that a system of UTU type is exponentially stable or else totally exponentially unstable. In other words if a given disturbance destabilizes the system it destabilizes it totally.

The system given in example (2.3) is not of UTU type however there exists many systems of UTU type. When a given system is unstable exponentially but not totally exponentially unstable (not of UTU type), the system is exponentially unstable on some regions but is exponentially stable on some others. Then can we characterize the regions on which the system is exponentially stable? Consequently can we characterize the UTU systems? The following theorem gives such a characterization:

Theorem 2.7. Assume that $A$ admits a system of orthonormal eigenfunctions $\left(\phi_{n}\right)_{n}$ associated to eigenvalues $\left(\lambda_{n}\right)_{n}$ and

$$
\begin{aligned}
& \text { (h1) } \operatorname{Re}\left(\lambda_{0}\right) \geqslant \operatorname{Re}\left(\lambda_{1}\right) \geqslant \ldots \geqslant \operatorname{Re}\left(\lambda_{n}\right) \geqslant \operatorname{Re}\left(\lambda_{n+1}\right) \geqslant \ldots \\
& (h 2) \lim _{n \rightarrow+\infty} \operatorname{Re}\left(\lambda_{n}\right)=-\infty
\end{aligned}
$$

Denote $\aleph=\left\{n: \operatorname{Re}\left(\lambda_{n}\right) \geq 0\right\}$ and $(1)$

$$
\Omega_{\mathrm{S}}=\Omega \quad \text { if } \aleph=\emptyset \quad \text { or } \quad \Omega_{\mathrm{S}}=\bigcap_{n \in \aleph}\left[\phi_{n}=0\right] \quad \text { if } \aleph \neq \emptyset
$$

Then for a given region $\sigma \subseteq \Omega$, with non null measure, the system is $\sigma$-exponentially stable if and only if

$$
\operatorname{meas}\left(\sigma \backslash \Omega_{\mathrm{S}}\right)=0
$$

\footnotetext{
${ }^{1}$ We denote $[f=0]=\{x / f(x)=0\}$ and $[f \neq 0]=\{x / f(x) \neq 0\}$.
} 
Proof: a) Suppose that $(S)$ is exponentially stable on $\sigma$.

If $\aleph=\emptyset$ then we obtain $\Omega_{\mathrm{S}}=\Omega$ and then meas $\left(\sigma \backslash \Omega_{\mathrm{S}}\right)=$ meas $(\sigma \cap \emptyset)=0$.

Now if $\aleph \neq \emptyset$ we know that there exists $\alpha>0$ and $M>0$ such that

$$
\left\|\chi_{\sigma} z(t)\right\| \leq M e^{-\alpha t}\left\|z_{0}\right\| \quad \forall t>0, \quad \forall z_{0} \in \mathrm{L}^{2}(\Omega)
$$

For all $n \in \aleph$ we have meas $\left(\sigma \cap\left[\phi_{n} \neq 0\right]\right)=0$, else $z_{0}=\phi_{n}$ gives $z(t)=e^{\lambda_{n} t} \phi_{n}$ and then

$$
e^{t \operatorname{Re}\left(\lambda_{n}\right)}\left\|\chi_{\sigma} \phi_{n}\right\| \leq M e^{-\alpha t}\left\|\phi_{n}\right\| \quad, \quad t>0
$$

But in this case $\left\|\chi_{\sigma} \phi_{n}\right\| \neq 0$ and (9) gives

$$
e^{\left(\operatorname{Re}\left(\lambda_{n}\right)+\alpha\right) t} \leq M \frac{\left\|\phi_{n}\right\|}{\left\|\chi_{\sigma} \phi_{n}\right\|} \quad, \quad t>0
$$

which is not satisfied when $t$ tends to infinity because $\operatorname{Re}\left(\lambda_{n}\right)+\alpha>0$.

Then meas $\left(\sigma \cap\left[\phi_{n} \neq 0\right]\right)=0, \forall n \in \aleph$, and then

$$
\begin{aligned}
\operatorname{meas}\left(\sigma \backslash \Omega_{\mathrm{S}}\right) & =\operatorname{meas}\left(\sigma \cap \Omega_{\mathrm{S}}^{\mathrm{c}}\right)=\operatorname{meas}\left(\sigma \cap\left(\bigcup_{n \in \aleph}\left[\phi_{n} \neq 0\right]\right)\right) \\
& =\operatorname{meas}\left(\bigcup_{n \in \aleph}\left(\sigma \cap\left[\phi_{n} \neq 0\right]\right)\right) \leq \sum_{n \in \aleph} \operatorname{meas}\left(\sigma \cap\left[\phi_{n} \neq 0\right]\right)=0
\end{aligned}
$$

b) Reciprocally let $\sigma$ be a given region with non null measure and satisfies (8).

If $\aleph=\emptyset$, the system is exponentially stable and then it is also $\sigma$-exponentially stable.

If $\aleph \neq \emptyset$, due to hypothesis $7, \aleph$ has a finite number of elements:

Denote $n_{1}=\inf \left(\aleph^{c}\right)$. Then $\aleph=\left\{0, \ldots n_{1}-1\right\}$ and

$$
\operatorname{Re}\left(\lambda_{n_{1}-1}\right) \geqslant 0>\operatorname{Re}\left(\lambda_{n_{1}}\right) \geqslant \ldots
$$

For every $n \in \aleph$ we have meas $\left(\sigma \cap\left[\phi_{n} \neq 0\right]\right) \leq \operatorname{meas}\left(\sigma \cap \Omega_{\mathrm{S}}^{\mathrm{c}}\right)=0$ and then $\chi_{\sigma} \phi_{n}=0$ almost $\partial$

$$
\chi_{\sigma} z_{\mathrm{I}}(t)=\sum_{n \in \aleph} e^{\lambda_{n} t}\left\langle z_{0}, \phi_{n}\right\rangle \chi_{\sigma} \phi_{n}=0
$$

We deduce that for all $z_{0} \in X$

$$
\begin{aligned}
\left\|\chi_{\sigma} z(t)\right\|^{2}=\left\|\chi_{\sigma} z_{\mathrm{S}}(t)\right\|^{2} \leqslant\left\|z_{\mathrm{S}}(t)\right\|^{2}= & \sum_{n \notin \aleph} e^{2 t \operatorname{Re}\left(\lambda_{n}\right)}\left\langle z_{0}, \phi_{n}\right\rangle^{2} \\
& \leq e^{2 t \operatorname{Re}\left(\lambda_{n_{1}}\right)} \sum_{n \notin \aleph}\left\langle z_{0}, \phi_{n}\right\rangle^{2} \leq e^{2 t \operatorname{Re}\left(\lambda_{n_{1}}\right)}\left\|z_{0}\right\|^{2}
\end{aligned}
$$

with $\operatorname{Re}\left(\lambda_{n_{1}}\right)<0$ and then the system is $\sigma$-exponentially stable. $\square$

Remark 2.8. The condition meas $\left(\sigma \backslash \Omega_{\mathrm{S}}\right)=0$ means that $\sigma \subseteq \Omega_{\mathrm{S}}$ almost everywhere ; That is almost all the region $\sigma$ is contained in $\Omega_{\mathrm{S}}$ (except a neglectable part).

We deduce from this theorem that when a given system is unstable (exponentially) the domain $\Omega$ can be divided on two regions: One region $\Omega_{\mathrm{S}}$ where the system is exponentially stable and another region $\Omega_{\mathrm{S}}^{\mathrm{c}}$ where the system is totally unstable. And consequently all region $\sigma$ is divided also on two regions: one region $\sigma \cap \Omega_{\mathrm{S}}$ 




$\aleph=\varnothing(\Omega$ is stable $)$

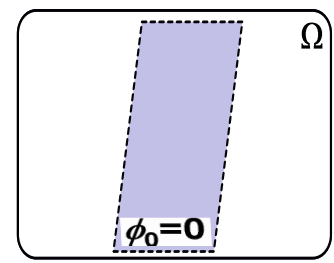

$\aleph=\{0\}$

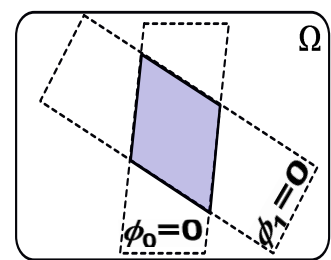

$\aleph=\{0,1\}$

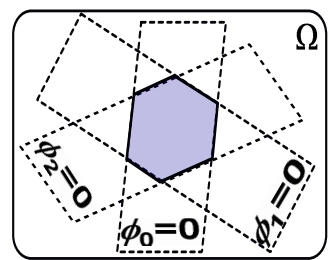

$\aleph=\{0,1,2\}$

FiguRE 1 . Zone of stability $\Omega_{\mathrm{S}}$ (grised zone) according to $\aleph$.

where the system is exponentially stable and another region $\sigma \cap \Omega_{\mathrm{S}}^{\mathrm{c}}$ where the system is totally unstable. The figure 1 illustrates the different situations.

An extreme case is when meas $\left(\Omega_{\mathrm{S}}^{\mathrm{c}}\right)=0$ (or $\Omega_{\mathrm{S}}^{\mathrm{c}}$ is empty) then the system is exponentially stable on all region with non null measure.

The other extreme case is when meas $\left(\Omega_{\mathrm{S}}\right)=0$ (or $\Omega_{\mathrm{S}}$ is empty), the system is then exponentially stable or else totally unstable. This is the case for systems of UTU type which are characterized by the following corollary:

Corollary 2.9. If $A$ admits a system of orthonormal eigenfunctions $\left(\phi_{n}\right)_{n}$ associated to eigenvalues $\left(\lambda_{n}\right)_{n}$ satisfying (7) then we have the equivalence:

(i) $(S)$ is of UTU type ;

(ii) meas $\left(\Omega_{\mathrm{S}}\right)=0$.

The corollary is deduced from theorem 2.7 .

Remark 2.10. If meas $\left[\phi_{n}=0\right]=0$ for all $n$ then the system is of UTU type but the converse is not true. It is the case for example if there exists two eigenfunctions $\phi_{n_{1}}$ and $\phi_{n_{2}}$ such that meas $\left[\phi_{n_{i}}=0\right] \neq 0$ for $i=1,2$ and meas $\left(\bigcap_{i=1,2}\left[\phi_{n_{i}}=0\right]\right)=0$ : the system is of UTU type.

Remark 2.11. For distributed parameter systems, characterized by their spatiotemporal evolution, the space variable plays an important role. When $A$ admits a system of orthonormal eigenfunctions $\left(\phi_{n}\right)_{n}$, associated to eigenvalues $\left(\lambda_{n}\right)_{n}$, the eigenfunctions $\phi_{n}(x)$ translates the effect of the eigenvalues $\lambda_{n}$ through the domain $\Omega$ (for exponential stability). This explains the fact that in the region $\sigma$ of $\Omega$ where $\phi_{n_{0}}(x)=0$, the effect (on stability) of the corresponding eigenvalue is not important in the sense that the system (S) can be $\sigma$-regionally exponentially stable either when $\Re\left(\lambda_{n_{0}}\right) \geq 0$ (see figure 1 ).

As an application of the theorem 2.7, corollary 2.9 and remark 2.10 we have the two following examples.

Example 2.12. Consider the system described by the following state equation in $\Omega=] 0,1[$

$$
\left\{\begin{array}{l}
\left.\frac{\partial z}{\partial t}=\alpha \frac{\partial^{2} z}{\partial x^{2}}+\beta \frac{\partial z}{\partial x} \quad \Omega \times\right] 0, T[ \\
z(0)=z_{0} \\
z(0, t)=z(1, t)=0
\end{array}\right.
$$

where $\alpha$ and $\beta$ are a non null real. 
Using corollary 2.9, we deduce that the system is of UTU type because the corresponding eigenfunctions are given by [4]

Note that the corresponding eigenvalues are given by [4]

$$
\phi_{n}(x)=\sqrt{2} \exp \left(-\frac{\beta x}{2 \alpha}\right) \sin (n \pi x)
$$

$$
\lambda_{n}=-\alpha n^{2} \pi^{2}-\frac{\beta^{2}}{4 \alpha}
$$

The system is totally unstable if $\alpha<0$ and exponentially stable if $\alpha>0$.

Example 2.13. Consider the Haar system defined on $\Omega=] 0,1[$ by [1]

$$
\begin{cases}\frac{\partial z(t)}{\partial t}=A z(t) & \Omega \times I \\ z(0) & \Omega\end{cases}
$$

with for all $z \in L^{2}(\Omega)$

$$
A z=\lambda_{1}\langle z, 1\rangle+\sum_{(n, m) \in \Lambda} \lambda_{(n, m)}\left\langle z, h_{(n, m)}\right\rangle h_{(n, m)}
$$

where $\Lambda=\left\{(n, m) \in \mathbb{N}^{2} / m<2^{n}\right\}$ and $\left(\lambda_{n, m}\right)_{(n, m) \in \Lambda}$ are real. $h_{(n, m)}$ are the Haar's functions defined for $(n, m) \in \Lambda$ and $x \in \Omega$ by

$$
h_{(n, m)}(x)=\left\{\begin{array}{ccc}
2^{n / 2} & \text { if } & x \in\left[\frac{m}{2^{n}}, \frac{m+\frac{1}{2}}{2^{n}}\right] \\
-2^{n / 2} & \text { if } & x \in\left[\frac{m+\frac{1}{2}}{2^{n}}, \frac{m+1}{2^{n}}\right] \\
0 & \text { elsewhere }
\end{array}\right.
$$

The family $\left\{1, h_{(n, m)} /(n, m) \in \Lambda\right\}$ is an orthonormal basis of $\mathrm{L}^{2}(\Omega)$.

We notice for this example that for the eigenvalue $\lambda_{1}$ the corresponding eigenfunction is $\phi_{1}(x)=1$ for $\left.x \in\right] 0,1[$ and for $\lambda_{0,0}$ the corresponding eigenfunction $h_{0,0}(x) \neq 0$ for all $\left.x \in \Omega=\right] 0,1[$. We consider the following case:

$$
\mid \begin{array}{lll}
\lambda_{1}=-\pi & \text { if } & n \geq 0 \\
\lambda_{n, 0}=-(n+2) \pi & \text { if } & n<3, \quad m \neq 0 \\
\lambda_{n, m}=-(n+m+1) \pi & \text { if } & n \geq 3 \text { and } m \neq 0 \\
\lambda_{n, m}=4-\sup (n, m) &
\end{array}
$$

Then we have:

Which gives:

$$
\lambda_{n, m}\left\{\begin{array}{ccc}
=1 & \text { if } & \sup (n, m)=3 \\
=0 & \text { if } & \sup (n, m)=4 \\
\leqslant-1 & \text { if } & \sup (n, m)<3 \text { or } \sup (n, m)>4
\end{array}\right.
$$

As

and

$$
\aleph=\{(3,1),(3,2),(3,3),(3,4),(4,1),(4,2),(4,3),(4,4)\}
$$

$$
\left.\left[h_{n, m}=0\right]=\right] 0, \frac{m}{2^{n}}[\cup] \frac{m+1}{2^{n}}, 1[
$$

$$
\left.\Omega_{S}=\bigcap_{(n, m) \in \aleph}\left[h_{n, m}=0\right]=\right] 0, \frac{1}{16}[\cup] \frac{5}{8}, 1[
$$

The system is not of UTU type and for all $\sigma \subset] 0, \frac{1}{16}[\cup] \frac{1}{2}, 1[$ the system is exponentially stable on $\sigma$. 


\section{Remark 2.14.}

(1) For systems of UTU type, there is equivalence between global and regional stability and consequently in this case the smallest disturbance that destabilizes the system destabilizes it totally (all regions are destabilized simultaneously).

(2) There exists systems which are not of UTU type (example (2.3) and example (2.13)). For such a systems we consider the regional stability radius problem.

\section{REGIONAL STABILITY RADIUS}

Consider the system given by the following state equation defined on $\Omega \subset \mathbb{R}^{n}$

$$
\left\{\begin{array}{l}
\dot{z}(t)=A z(t)+B u(t) \quad t>0 \\
z(0)=z_{0}
\end{array}\right.
$$

Let $\sigma$ be a subset of $\Omega$ with non null measure.

As we are interested by the state on the region $\sigma$, we consider the regional output function given by :

$$
y^{\sigma}(t)=C \chi_{\sigma}^{*} \chi_{\sigma} z(t)
$$

which can be written as follow: $y^{\sigma}(t)=C_{\sigma} z(t)$ where $\chi_{\sigma}^{*}$ is adjoint operator of $\chi_{\sigma}$ and $C_{\sigma}: \psi \in L^{2}(\Omega) \rightarrow$ $C \chi_{\sigma}^{*} \chi_{\sigma} \psi \in Y$ and $C$ is given in (2).

We assume that $\left(A, B, C_{\sigma}\right)$ is in the Pritchard Salamon class.

The solution of the disturbed system, in the case where $u(t)=D y^{\sigma}(t)$, where $D \in \mathcal{D}$ and $\mathcal{D}$ is a subset of $\mathcal{L}\left(L_{Y}^{2}, L_{U}^{2}\right)$, is given by:

$$
z(t)=S(t) z_{0}+\int_{0}^{t} S(t-s) B D C_{\sigma} z(s) \mathrm{d} s
$$

and the observation of the solution $z(t)$ on $\sigma$ is given by

$$
y^{\sigma}(t)=C_{\sigma} z(t)=C_{\sigma} S(t) z_{0}+C_{\sigma} \int_{0}^{t} S(t-s) B D C_{\sigma} z(s) \mathrm{d} s
$$

Which gives

where $y_{0}^{\sigma}(t)=C_{\sigma} S(t) z_{0}$ and

$$
y^{\sigma}=y_{0}^{\sigma}+C_{\sigma} L D y^{\sigma}
$$

is defined by

$$
L: \mathrm{L}^{2}(0, \infty ; U) \rightarrow \mathrm{L}^{2}(0, \infty ; X)
$$

We have the following definition

$$
(L u)(t)=\int_{0}^{t} S(t-s) B u(s) \mathrm{d} s
$$

Definition 3.1. The regional stability radius (of $\sigma$ ) of (18) is

$$
\hat{\rho}_{\sigma}\left(L, C_{\sigma}, \mathcal{D}\right)=\sup \left\{d ; r<d, \text { implied } \exists K_{r}\right.
$$

We have the following result 
Theorem 3.2. We have

$$
\widehat{\rho}_{\sigma}\left(L, C_{\sigma}, \mathcal{L}\left(\mathrm{L}_{Y}^{2}, \mathrm{~L}_{U}^{2}\right)\right)=\left\|C_{\sigma} L\right\|^{-1}
$$

Proof. The principle of the proof is the same as in Pritchard and Townley [7], but with taking into account the regional aspect. Indeed, if $\|D\|<\left\|C_{\sigma} L\right\|^{-1}$, then $\left\|C_{\sigma} L D\right\|<1$ and then $C_{\sigma} L D$ is a contraction on $L_{Y}^{2}$ and then (18) admits a unique solution $y^{\sigma}(.) \in \mathrm{L}_{Y}^{2}$. Consider now a sequence $u_{n}^{\sigma}$ in $L_{U}^{2}$ such that $\left\|u_{n}^{\sigma}\right\|=1$ and $\left\|C_{\sigma} L u_{n}^{\sigma}\right\|=\mu_{n}^{\sigma}$ is increasing to $\mu^{\sigma}=\left\|C_{\sigma} L\right\|$. Let $h_{n}^{\sigma}=\left(\mu^{\sigma}\right)^{-1} C_{\sigma} L u_{n}^{\sigma}$ and $y_{n}^{\sigma}=y_{0}^{\sigma}+\alpha_{n}^{\sigma} h_{n}^{\sigma}$ where

$$
\alpha_{n}^{\sigma}=\left[1-\left\|h_{n}^{\sigma}\right\|^{2}\right]^{-1}\left\{\left\langle y_{0}^{\sigma}, h_{n}^{\sigma}\right\rangle+\left[\left\langle y_{0}^{\sigma}, h_{n}^{\sigma}\right\rangle^{2}+\left(1-\left\|h_{n}^{\sigma}\right\|^{2}\right)\left\|y_{0}^{\sigma}\right\|^{2}\right]^{\frac{1}{2}}\right\}
$$

Then we have $\left\|y_{n}^{\sigma}\right\|=\alpha_{n}$ and $y_{n}^{\sigma} \in L_{Y}^{2}$ for each $n$.Put $D_{n} y=\left(u_{n}^{\sigma}\left\langle y_{n}^{\sigma}, y^{\sigma}\right\rangle\right) /\left(\mu^{\sigma}\left\|y_{n}^{\sigma}\right\|\right)$, then

(i) $\left\|D_{n}\right\|=\left\|C_{\sigma} L\right\|^{-1}$ and (ii) $\left\|C_{\sigma} L D_{n}\right\|=\frac{\mu_{n}^{\sigma}}{\mu^{\sigma}}<1$.

Then for each $n, y_{n}^{\sigma}$ is the unique solution of $y^{\sigma}=y_{0}^{\sigma}+C_{\sigma} L D_{n} y^{\sigma}$. As $h_{n}^{\sigma}=\left(\mu^{\sigma}\right)^{-1} L u_{n}^{\sigma}$, we choose $u_{n}^{\sigma}$ such that $\left\langle y_{0}^{\sigma}, h_{n}^{\sigma}\right\rangle \geq 0$.

And then from (20), we obtain $\left\|y_{n}^{\sigma}\right\| \rightarrow \infty$.

Definition 3.3. The regional stability radius of $\sigma$, relatively to $\mathcal{D} \subset \mathcal{L}\left(L_{Y}^{2}, L_{U}^{2}\right)$, is the greatest positive real $\mathrm{r}$, denoted $r_{\sigma}\left(A, B, C_{\sigma}, \mathcal{D}\right)$, such that for all $D \in \mathcal{D}$ the solution of (16) is $\sigma$-exponentielly stable for each $z_{0}$ in $X$.

The regional stability radius of $\sigma$ is the greatest positive real $\mathrm{r}$, noted $r_{\sigma}\left(A, B, C_{\sigma}\right)$, such that for all $D \in \mathcal{L}$ $(Y, U),\|D\|<r$ the solution of (16) is $\sigma$-exponentielly stable for each $z_{0}$ in $X$.

That means that regional stability radius of $\sigma$ (respectively relatively to $\mathcal{D}$ ) is the smallest disturbance (relatively in $\mathcal{D}$ ) that destabilizes the system on the region $\sigma$.

For systems which aren't of UTU we can also define the total stability radius as follow

Definition 3.4. The total stability radius of system $(S)$, relatively to $\mathcal{D} \subset \mathcal{L}\left(L_{Y}^{2}, L_{U}^{2}\right)$, is the greatest positive real $r$, noted $r_{\text {total }}^{\mathcal{D}}$, such that for all $D \in \mathcal{D},\|D\|<r$, the solution of (16) (for each $z_{0}$ in $X$ ) is not totally exponentially unstable.

The total stability radius of system $(S)$ is the greatest positive real $\mathrm{r}$, noted $r_{\text {total }}^{\mathcal{D}}$, such that for all $D \in$ $\mathcal{L}\left(L_{Y}^{2}, L_{U}^{2}\right),\|D\|<r$ the solution of (16) (for each $z_{0}$ on $X$ ) is not totally exponentially unstable.

That means that the total stability radius is the smallest disturbance which destabilizes totally the system. We have the result

Proposition 3.5. If we denote $r_{\Omega}$ the global stability radius, then:

1. For all $\sigma \subset \Omega$, meas $(\sigma) \neq 0$, we have $r_{\Omega} \leq r_{\sigma} \leq r_{\text {total }}$.

2. If the system is of UTU type, then $\forall \sigma \subset \Omega$, meas $(\sigma) \neq 0, r_{\Omega}=r_{\sigma}=r_{\text {total }}$.

We have the same results for stability radius relatively to a given subset $\mathcal{D}$.

The proof lies with the fact that regional instability implies global instability and we have equivalence for the UTU systems.

To illustrate the regional stability radius we consider the following examples

\section{EXAMPLES}

In this section we consider the three examples given bellow (example 2.3, 2.12 and 2.13). We begin by example (2.12). 
Example 4.1. Consider the system described by the following state equation in $\Omega=] 0,1[$

$$
\left\{\begin{array}{l}
\left.\frac{\partial z}{\partial t}=\alpha \frac{\partial^{2} z}{\partial x^{2}}+\beta \frac{\partial z}{\partial x}+B u ; \Omega \times\right] 0, T[ \\
z(0)=z_{0} \\
z(0, t)=z(1, t)=0
\end{array}\right.
$$

Augmented by the output function

$$
y(t)=C z(t)
$$

where $\alpha$ and $\beta$ are a non null real.

In the autonomous case, $u=0$, the system is of UTU type because the corresponding eigenfunctions are [4]

$$
\phi_{n}(x)=\sqrt{2} \exp \left(-\frac{\beta x}{2 \alpha}\right) \sin (n \pi x)
$$

Remark that the corresponding eigenvalue are given by [4]

$$
\lambda_{n}=-\alpha n^{2} \pi^{2}-\frac{\beta^{2}}{4 \alpha}
$$

Consider the case where the autonomous system is exponentially stable i. e. $\alpha>0$ and let us determine the stability radius of system (21) in two cases:

Case 1. Stability radius of system (21) relatively to a particular subset $\mathcal{D}$ of $\mathcal{L}\left(L_{Y}^{2}, L_{U}^{2}\right)$ for $B=I$ and $C=I$. We consider

$$
\mathcal{D}=\left\{D_{\gamma} \in \mathcal{L}\left(L_{Y}^{2}, L_{U}^{2}\right) \text { defined by } D_{\gamma} y=\gamma y: \gamma \in \mathbb{R} \quad D_{\gamma}=\gamma I\right\}
$$

The disturbed system is then given by

$$
\left\{\begin{array}{l}
\left.\frac{\partial z}{\partial t}=\alpha \frac{\partial^{2} z}{\partial x^{2}}+\beta \frac{\partial z}{\partial x}+\gamma z \quad \Omega \times\right] 0, T[ \\
z(0)=z_{0} \\
z(0, t)=z(1, t)=0
\end{array}\right.
$$

The corresponding eigenfunction are given by [4]

$$
\phi_{n}(x)=\sqrt{2} \exp \left(-\frac{\beta x}{2 \alpha}\right) \sin (n \pi x)
$$

The nature of the corresponding eigenfunctions means that the system is of UTU type and consequently the total and regional stability radius are the same.

The corresponding eigenvalue are given by [4]

$$
\lambda_{n}=\gamma-\alpha n^{2} \pi^{2}-\frac{\beta^{2}}{4 \alpha}
$$

We have

$$
r(A, I, I, \mathcal{D})=\alpha \pi^{2}+\frac{\beta^{2}}{4 \alpha}
$$


Case 2. We consider now the particular case where $\alpha=-1, \beta=0, B u=\delta(x-b) u$ and $y(t)=C z=\left\langle z, \phi_{n_{0}}\right\rangle$, we obtain [4]

$$
G(i \omega) u=\left\langle\sum_{n} \frac{\phi_{n}(b) u}{i \omega-\lambda_{n}} \phi_{n}, \phi_{n_{0}}\right\rangle=\frac{\phi_{n_{0}}(b)}{i \omega-\lambda_{n_{0}}} u
$$

Which gives for $b$ such that $\phi_{n_{0}}(b) \neq 0$,

$$
r(A, B, C)=\frac{\left|\lambda_{n_{0}}\right|}{\left|\phi_{n_{0}}(b)\right|}
$$

As an example for $b=\frac{1+2 m}{2 n_{0}}, r(A, B, C)=\frac{n_{0}^{2} \pi^{2}}{\sqrt{2}}$.

For more details about the computation of the stability radius (and transfer functions) for different systems see Bernoussi [4].

Remark 4.2. As for the first case, the smallest disturbance that destabilizes the system destabilizes it totally (the system is of UTU type) and depends on the location (space) of the actuator but it depends also of the eigenfunction $\lambda_{n_{0}}$ for which the corresponding eigenfunction $\phi_{n_{0}}(x)=\sqrt{2} \sin \left(n_{0} \pi x\right)\left(\operatorname{meas}\left\{\phi_{n_{0}}(x)=0\right\}=0\right)$. In the first case we have considered through $C=I$ all the eigenvalues $\lambda_{n}$ (eigenfunctions $\phi_{n}$ ) but in the second case we have considered just $\lambda_{n_{0}}$ (eigenfunctions $\phi_{n_{0}}$ ).

We consider now an example where the operator $A$ admits an orthonormal system of eigenfunctions but the system is not of UTU type: Example (2.13).

Example 4.3. Consider the Haar system given in example (2.13) but excited by a pointwise actuator located at point $b \in] 0,1[$

$$
\begin{cases}\frac{\partial z}{\partial t}=A z(t) & +B u(t) \quad \Omega \times I \\ z(0)=z_{0} & \Omega\end{cases}
$$

With for all $z \in \mathrm{L}^{2}(\Omega)$,

$$
A z=\lambda_{1}\langle z, 1\rangle+\sum_{(n, m) \in \Lambda} \lambda_{n, m}\left\langle z, h_{(n, m)}\right\rangle h_{(n, m)}
$$

where $\Lambda=\left\{(n, m) \in \mathbb{N}^{2} / m<2^{n}\right\}$ and $\left(\lambda_{n, m}\right)_{(n, m) \in \Lambda}$ are reals.

We consider the case where $(B=I, C=I)$ and $\mathcal{D}$ given by $(24)$. The disturbed system is given by

$$
\left\{\begin{array}{l}
\frac{\partial z}{\partial t}=A z(t)+\gamma z(t) \quad \Omega \times I \\
z(0)=z_{0} \Omega
\end{array}\right.
$$

In this case the corresponding eigenfunctions are 1 and $h_{(n, m)}$ given by (13) and the corresponding eigenvalues are $\lambda_{1}+\gamma$ and $\lambda_{n, m}+\gamma$. We have the result

Proposition 4.4. 1. The stability radius relatively to $\mathcal{D}$ is $r(A, I, I, \mathcal{D})=\min \left(\left|\lambda_{1}\right|,\left|\lambda_{n, m}\right|\right)$

2. The total stability radius $r_{\text {total }}(A, I, I, \mathcal{D})=\left|\lambda_{1}\right|$.

In the two examples given above we have considered the case where the operator $A$ admits a system of orthonormal eigenfunction $\phi_{n}(x)$ associated to eigenvalues $\lambda_{n}$. In the following example we consider another example. 
Example 4.5. Consider the system given in example (2.3) but excited by control $B u$.

$$
\left\{\begin{array}{l}
\dot{z}(t)=a(x) z(t)+B u(t) \quad t>0 \\
z(0)=z_{0}
\end{array}\right.
$$

Augmented by the output function

$$
y(t)=C z(t)
$$

We consider $\Omega=] 0,4[, a(x)=x-5, B=I$ and $C=I$.

Remark that for the autonomous system $(u=0),(31)$ is exponentially stable. For $u=D y$ the system (31) becomes:

$$
(S)\left\{\begin{array}{l}
\dot{z}(t)=[(x-5)+D] z(t) \quad 0<t<T \\
z(0)=z_{0}
\end{array}\right.
$$

The system (33) will be stable (on all $\Omega$ ) for all $D$ such that: $|D|<1$ and the system isn't exponentially stable for $D=1$. So $r_{\Omega}(A, B, C)=1$.

Analysis of regional aspect. We remark at first that the system $(S)$ isn't of UTU type. $(S)$ is exponentially stable on the region where $x-5+D<0$.

Consider now the three following regions: $\sigma_{1}=[0,1], \sigma_{2}=[1,2]$ and $\sigma_{3}=[2,4]$, then:

- For $\sigma_{3}$ : for $D$ such that $|D|<1$ the system is $\sigma_{3}$ exponentially stable but for $D=1$ the system isn't $\sigma_{3}$ exponentially stable. So the regional stability radius of $\sigma_{3}$ is $r_{\sigma_{3}}=1$.

- For $\sigma_{2}$ : for $D$ such that $|D|<3$ the system is $\sigma_{2}$ exponentially stable but for $D=3$ the system isn't $\sigma_{2}$ exponentially stable. So the regional stability radius of $\sigma_{2}$ is $r_{\sigma_{2}}=3$.

- For $\sigma_{1}$ : for $D$ such that $|D|<4$ the system is $\sigma_{1}$ exponentially stable but for $D=4$ the system isn't $\sigma_{1}$ exponentially stable. So the regional stability radius of $\sigma_{1}$ is $r_{\sigma_{1}}=4$.

\section{Remark 4.6.}

(1) The smallest disturbance which destabilizes the system $(S)$ (globally) is $D=1$ and the smallest disturbance which destabilizes it totally is $D=5$ and for all region $\sigma \subset \Omega$ with meas $(\sigma) \neq 0$, we have $1 \leq r_{\sigma} \leq 5$. Remark that $(S)$ isn't of UTU type.

(2) For systems of UTU type we have equality: The smallest disturbance which destabilize the system globally destabilize it totally (destabilizes all region $\sigma \subset \Omega$ with meas $(\sigma) \neq 0$ ).

(3) We can find this results also by considering in (32) as a measure function $y^{\sigma_{i}}=C \chi_{\sigma_{i}} z$ for $i=1,2$ and 3 .

This example shows clearly the importance of regional stability radius for systems which are not of UTU type.

\section{CONCLUSION}

In this work we have considered, for distributed parameter systems, the problem of robust regional stability and particularly that of regional stability radius. We have proved the important role that plays the space variable for such a systems. As the stability radius is the "smallest disturbance" which destabilizes a given exponentially stable system, we have considered the problem of "instability" through the space variable and consequently we have introduced the UTU (Unstable/Totally Unstable) systems. For UTU systems, whose instability is equivalent to the total instability, the stability radius is the same for all region $\sigma \subset \Omega$. However 
we have shown that there exists some systems which are not of UTU type (for which instability (global) is not equivalent to regional instability), and for which each zone $\sigma \subset \Omega$ has its proper stability radius which could be calculated by a "regional" measure. For such a systems it would be interesting to develop all the other problems of robust control through a regional aspect. For example as each zone $\sigma \subset \Omega$ has its own stability radius we can introduce the concept of vulnerability $[3,5,17]$ to stability (A given zone $\sigma_{1}$ will be "more vulnerable to stability" than $\sigma_{2}$ if its stability radius $\left.r_{\sigma_{1}} \leq r_{\sigma_{2}}\right)$. Consequently we can consider problems of robust control

(optimal) through the "more" vulnerable zone. Also it will be very interesting to consider time varying and non linear systems. Some of those problems are under investigation.

\section{REFERENCES}

[1] L. Afifi, A. El Jai and E. Zerrik, 2008. Systèmes dynamiques 2 : Analyse régionale des systèmes linéaires distribués. Collection études. Presse Universitaire de Perpignan.

[2] A. Bernoussi, 2009. Regional stability radius of distributed parameter systems. Proceeding Intenational confrence Systems Theory: Modelling, analysis and control. pp. 201-208. Collection études. Presse Universitaire de Perpignan.

[3] A. Bernoussi, 2007. Spreadability and vulnerability of distributed parameter systems. International Journal of Systems Science, Vol. 38, N. 4, April 2007, 305-317.

[4] A. Bernoussi, 1993. Approche Fréquentielle des systèmes Distribués - Relation avec les capteurs et les actionneurs. Thèse de Doctorat, Université de Perpignan, France.

[5] A. Bernoussi et M. Amharref, 2003. Etalabilité - vulnérabilité. Annals of University of Craiova, Math. Comp. Sci. Ser. Volume 30, 2003, pp 53-62. ISSN 1223-6934.

[6] W. Desch and W. Shappacher, 1985. Spectral properties of finite dimensional perturbed linear semigroups. J. Differential Equations 59 (1985), 80-102.

[7] A. J. Pritchard and S. Townley, 1989. Robustness of linear Systems. Journal of Differentiel Equations. Vol. 77, No. 2, February 1989.

[8] A. J. Pritchard and S. Townley, 1986. A stability radius for infinite dimensional systems. In "Infinite dimensional systems. Proc. Vorau" (F. Kappel, Ed), pp. 272-291. Springer-Verlag, New York/Berlin, 1986.

[9] D. Hinrichsen and A. J. Pritchard, 1986. Stability radii for linear systems. Systems control lett. 7, pp. 1-10.

[10] D. Hinrichsen and A. J. Pritchard, 1986. Stability radius forstructured perturbations and the algebraic Riccati equation. Systems Control lett. 8, No. 2, 105-113.

[11] A. El Jai, 2004. Elément d'Analyse et de Contrôle des systèmes. Collection Etudes, Presses Universitaires de Perpignan, ISBN: 2-914518-60-9.

[12] A. E. Jai, M. C. Simon, and E. Zerrik, 1993. Regional observability and sensors structures. International Journal of Sensors and Actuators 39, 1993.

[13] A. E. Jai, M. C. Simon, E. Zerrik, and A. J. Pritchard. 1995. Regional controllability of distributed parameter systems. International Journal of Control 62, 1995. 43.

[14] R. Al-Saphory and A. E. Jai, 2002. Asymptotic regional state reconstruction. International Journal of Systems Sciences 33(13), pp. 1025-1037, 2002. 72.

[15] J. L. Lions, 1981 Some methods in the mathematical analysis of systems and their control. Sciences Press. Beijing. China.

[16] J. L. Lions and E. Magenes Probl'emes aux limites non homogènes et applications. Dunod. Paris. 1968.

[17] Y. Qaraai, A. Bernoussi and A. El Jai, 2007. How to control a spreadind disturbance for a class of non linear systems. Int. J. Appl. Math. Comput. Sci., 2008, Vol. 18, No. 2, 171-187. DOI: 10.2478/v10006-008-0016-9.

[18] Zerrik, E. \& Ouzahra, M. (2003). Regional stabilization for infinite-dimensional systems. International Journal of Control, $76(1), 73-81$. 